\title{
Neoadjuvant intraperitoneal chemotherapy for advanced stage gastric cancer (Review)
}

\author{
NICOLAE BACALBASA ${ }^{1,2}$, CAMELIA DIACONU ${ }^{3,4}$, BOGDAN SOCEA $^{5,6}$, FLORENTINA GHERGHICEANU ${ }^{7}$, \\ CORNEL SAVU $^{8,9}$, MIHAI DIMITRIU ${ }^{1,10}$, IRINA BALESCU ${ }^{11}$ and IOAN $\operatorname{CORDOS}^{8,9}$ \\ ${ }^{1}$ Department of Obstetrics and Gynecology, 'Carol Davila' University of Medicine and Pharmacy, 020021 Bucharest; \\ ${ }^{2}$ Department of Visceral Surgery, Center of Excellence in Translational Medicine 'Fundeni' Clinical Institute, \\ 022328 Bucharest; ${ }^{3}$ Department of Internal Medicine, 'Carol Davila' University of Medicine and Pharmacy, \\ 020021 Bucharest; ${ }^{4}$ Department of Internal Medicine, Clinical Emergency Hospital of Bucharest, 014461 Bucharest; \\ ${ }^{5}$ Department of Surgery, 'Carol Davila' University of Medicine and Pharmacy, 020021 Bucharest; ${ }^{6}$ Department of Surgery, \\ 'Sf. Pantelimon' Emergency Hospital, 33094 Bucharest; ${ }^{7}$ Department of Marketing and Medical Technology, \\ 'Carol Davila' University of Medicine and Pharmacy, 020021 Bucharest; ${ }^{8}$ Department of Thoracic Surgery, \\ 'Marius Nasta' National Institute of Pneumology, 050159 Bucharest; ${ }^{9}$ Department of Thoracic Surgery, \\ 'Carol Davila' University of Medicine and Pharmacy, 020021 Bucharest; ${ }^{10}$ Department of Obstetrics and Gynecology, \\ 'Sf. Pantelimon' Emergency Hospital, 33094 Bucharest; ${ }^{11}$ Department of Surgery, \\ 'Ponderas' Academic Hospital, 021188 Bucharest, Romania
}

Received June 8, 2021; Accepted July 8, 2021

DOI: $10.3892 / \mathrm{etm} .2021 .10749$

\begin{abstract}
Gastric cancer remains one of the most lethal malignancies especially when diagnosed in advanced stages of the disease; most often patients diagnosed later during the progression of their disease will present a certain degree of peritoneal contamination such as positive peritoneal cytology or peritoneal metastatic nodules. In such cases most often they then progress to peritoneal carcinomatosis and succumb to the disease within one year. In order to increase the lifespan in such cases multiple therapeutic strategies have been proposed such as radical surgery and intraperitoneal heated chemotherapy or direct intraperitoneal chemotherapy followed by radical surgery. To date, the benefits of intraperitoneal heated chemotherapy at the time of resection have been widely investigated; however the method is still associated with increased rates of perioperative complications. Therefore, attention was focused on investigating the benefits of such procedures as neoadjuvant therapies followed by radical surgery. The aim of the present review was to examine the most efficient therapeutic strategies in advanced-stage gastric cancer such as neoadjuvant laparoscopic heated intraperitoneal chemotherapy, perioperative heated intraperitoneal chemotherapy and neoadjuvant systemic and peritoneal chemotherapy.
\end{abstract}

Correspondence to: Dr Nicolae Bacalbasa, Department of Obstetrics and Gynecology, 'Carol Davila' University of Medicine and Pharmacy, 8 Eroii Sanitari Boulevard, 020021 Bucharest, Romania E-mail: nicolae_bacalbasa@yahoo.ro

Key words: gastric cancer, peritoneal lesions, intraperitoneal chemotherapy, morbidity, survival

\section{Contents}

1. Introduction

2. Methods

3. Principle of neoadjuvant HIPEC

4. Effectiveness of HIPEC in the neoadjuvant setting of peritoneal carcinomatosis from gastric cancer

5. Conclusions

\section{Introduction}

Gastric cancer represents one of the most lethal digestive malignancies affecting people worldwide especially in cases diagnosed in advanced stages of the disease; the overall estimated 5-year survival rate is $25 \%$ while in cases presenting advanced-stage disease this value significantly decreases to $<5 \%$ (1-3). As numerous other malignancies, gastric cancer may spread via multiple pathways such as the hematogenous one leading to the apparition of distant, visceral metastases, the lymphatic one leading to the development of lymph node metastases and the peritoneal one leading to the development of peritoneal carcinomatosis (1). This latter pathway of spread is caused by the exfoliation of malignant cells from the gastric serosa and further implantation of these cells at the level of the peritoneal cavity. In time these cells will proliferate and will progress to the development of peritoneal carcinomatosis nodules; it is estimated that up to $45 \%$ of patients present synchronous peritoneal nodules at the time of the initial diagnosis, while a similar proportion will develop such lesions at a certain stage of the disease even if radical surgery has been performed at the time of the initial diagnosis (1-3). Once peritoneal nodules of carcinomatosis develop, multiple 
complications such as ascites, bowel occlusion, bowel perforation or nutritional deprivation may develop, leading to the apparition of intractable complications and finally patients succumb to the disease (1). Therefore, previous studies have considered peritoneal carcinomatosis as a locoregional disease and not a systemic one. However, the estimated survival rate of patients presenting peritoneal carcinomatosis from gastric origin is $<6$ months, this lifespan being minimally improved in cases submitted to systemic chemotherapy $(4,5)$.

In this respect, aggressive surgical procedures such as intraperitoneal chemotherapy, early postoperative intraperitoneal chemotherapy, neoadjuvant intraperitoneal and systemic therapy and cytoreductive surgery have been proposed $(1,6-8)$. Hyperthermic intraperitoneal chemotherapy (HIPEC) has been demonstrated to play a significant role in the setting of advanced-stage gastric cancer as a prophylactic tool as well as in selected cases presenting peritoneal carcinomatosis (9-12). The method appears to be associated with several advantages such as direct delivery of high amounts of cytotoxic agents at the tumor level, use of heated agents which increase the antitumor effect and diminish systemic toxicity; all these advantages present significant interest especially in gastric cancer patients, in which disease recurs most frequently via the peritoneal route (13-15). However, the method has been demonstrated to be associated with significant rates of perioperative morbidity; therefore, attention was focused on identifying other therapeutic approaches which may maximize the effect of these procedures while also minimizing the risk of perioperative morbidity and mortality (1).

\section{Methods}

The present article is a literature review conducted on studies identified after searching the following keywords on Pubmed: 'neoadjuvant chemotherapy', 'advanced stage gastric cancer', 'peritoneal carcinomatosis from gastric cancer', 'hyperthermic chemotherapy'. Initially, 83 studies were identified from which studies in which the full text was not available, studies written in other languages other than English as well as case reports were excluded. Finally, 35 studies were identified, which were analyzed when writing the present review; the reviewed papers were published between 1995-2021.

\section{Principle of neoadjuvant HIPEC}

One of the most widely investigated therapeutic strategies is represented by using HIPEC as a neoadjuvant procedure, followed by interval debulking surgery. The method, performed in a laparoscopic manner appears to be associated with complete disappearance of the peritoneal metastases in up to $25 \%$ of cases and increased overall survival rates $(13,16)$. Therefore, minimally invasive surgery in association with neoadjuvant HIPEC alone (in the absence of debulking surgery) appears to maximize the oncological benefits and to minimize the risks of perioperative morbidity. In addition, other authors have also examined the possibility of performing neoadjuvant HIPEC as prophylaxis of peritoneal carcinomatosis in patients with positive cytology; in this respect, in cases presenting serosal invasion, peritoneal cytology should be retrieved and whenever malignant cells are encountered, neoadjuvant HIPEC followed by interval radical gastrectomy should be the option of choice $(17,18)$. However, an interesting difference of opinion should be underlined between Eastern and Western countries; while in Asian trial studies published thus far the effectiveness of HIPEC was demonstrated in patients with serosal invasion as well as in those with peritoneal carcinomatosis, in Western countries it appears that the method proved to be particularly efficient only in cases with serosal invasion. The difference may be explained through the fact that there are significant differences between the two types of tumors in regard to tumor biology, genetics and type of treatment $(19,20)$.

Another interesting concept is the one of preventive perioperative HIPEC in patients diagnosed with advanced stage gastric cancer; according to Sugarbaker (21), whenever a patient with advanced stage gastric cancer (with serosal invasion) is submitted to surgery an increased risk of intraoperative peritoneal contamination occurs via multiple mechanisms, therefore, surgical manipulation of the specimen, lymphatic transection during lymph node dissection as well as intraoperative venous hemorrhage may predispose to peritoneal seeding. Whenever a lymphatic is transected and its content is contaminated with malignant cells or a vein is sectioned and venous blood with possible tumor emboli reaches the peritoneal surface the risk of peritoneal contamination increases. Meanwhile, during the early postoperative period development of adherent syndromes and fibrin entrapment will fix the neoplastic cells at the level of the peritoneal surface and will further favor the development of peritoneal carcinomatosis. In this respect, Sugarbaker (21) proposed a prevention perioperative HIPEC protocol in all cases diagnosed with advanced stage gastric cancer.

\section{Effectiveness of HIPEC in the neoadjuvant setting of peritoneal carcinomatosis from gastric cancer}

The concept of neoadjuvant intraperitoneal chemotherapy has been initially taken into consideration in the absence of hyperthermia but in association with systemic chemotherapy. The method, also known as neoadjuvant intraperitoneal and systemic chemotherapy (NIPS), has been demonstrated to combine the benefits of the two pathways of administration of the cytotoxic drugs; therefore, a higher amount of chemotherapeutic agents will be concentrated at the level of the peritoneal nodules, reaching these lesions via hematogenous flow and also, directly via the peritoneal pathway. According to Sugarbaker, the most appropriate candidates for this therapeutic strategy are patients younger than 65 years old, with a good general status (Eastern Clinical Oncology Group score of two or less), no hematogenous or lymphatic metastases, normal hematogenous, renal, cardiac and hepatic function, no other synchronous malignancies and confirmed peritoneal contamination by cytology or histology (21). The procedure can be applied for 4 to 6 cycles and is further followed by radical surgery in cases in which a positive response (defined by negative cytology and regression of the peritoneal lesions) is achieved (21).

The role of neoadjuvant HIPEC in peritoneal carcinomatosis from gastric cancer has been thoroughly investigated thus far. It appears that the method plays a crucial role in cases presenting extended peritoneal lesions from the time of the 
initial diagnosis; therefore, performing two to five sequences of neoadjuvant HIPEC appears to significantly decrease the extent of the peritoneal lesions [quantified by the peritoneal carcinomatosis index (PCI)] and therefore to increase the rates of complete cytoreduction. One of the most eloquent studies which demonstrated the efficacy of neoadjuvant HIPEC in order to decrease the PCI and to increase the lifespan has been recently published by $\mathrm{Yu}$ et al (22); the study included 38 patients submitted to neoadjuvant systemic chemotherapy, HIPEC and debulking surgery (18 cases, conversion therapy group) and their outcomes were compared with the ones reported in the control group (20 patients submitted to chemotherapy and HIPEC solely). The authors reported a significant increase of the median overall survival in the conversion therapy group (21,1 months) when compared with the control group (in which the median overall survival rate was only 10,8 months $(\mathrm{P}=0.002)$. In addition, the authors underlined the fact that cases in which the PCI decreased under a value of 6 at the time of the second laparoscopy reported a significantly improved outcome when compared with those in which this value was higher (22).

Data published to date, as well as the ongoing clinical trials at this time aim to demonstrate the effectiveness of neoadjuvant HIPEC alone or in association with neoadjuvant systemic chemotherapy in patients with positive cytology or limited peritoneal lesions (23). According to Beeharry et al, patients with positive cytology and in the absence of macroscopically visible peritoneal lesions during laparoscopy should be further submitted to neoadjuvant HIPEC while cases presenting macroscopically visible but limited lesions should be further submitted to NIPS (23). The same study group elaborated a protocol and a clinical trial in which they aimed to compare the efficacy of neoadjuvant laparoscopic HIPEC in association with neoadjuvant systemic chemotherapy followed by radical gastrectomy and HIPEC vs. radical gastrectomy followed by adjuvant chemotherapy in advanced stage gastric cancer. The multicentric phase III randomized controlled trial included 326 patients who were randomized to one of the two groups after laparoscopic exploration. Cases included in the first arm received one procedure of neoadjuvant laparoscopic HIPEC (60 min at $43^{\circ} \mathrm{C}$ with $80 \mathrm{mg} / \mathrm{m}^{2}$ paclitaxel) followed by three cycles of neoadjuvant systemic chemotherapy with oxaliplatin, radical D2 gastrectomy with intraoperative HIPEC and another 5 cycles of oxaliplatin-based adjuvant chemotherapy. The control group was submitted to standard radical D2 gastrectomy followed by 8 cycles of adjuvant systemic chemotherapy with oxaliplatin. The aim of the study was to compare the long-term outcomes between the two arms, defined by the 5-year progression-free survival rate, the 5-year overall survival rate and the peritoneal metastasis rate as well as the short-term outcomes defined by the overall morbidity rate (24).

The efficacy and safety of neoadjuvant HIPEC in patients with positive peritoneal cytology as well as in cases with radiologically occult peritoneal carcinomatosis after neoadjuvant chemotherapy or even chemoradiotherapy has been demonstrated by Badgwell et al in 2017 (25). The study was conducted on 19 patients; peritoneal carcinomatosis being present at the time of the initial diagnosis in 13 cases and positive cytology in the remaining 6 cases. These cases were submitted to 38 laparoscopic HIPEC procedures which were performed after a median number of 8 cycles of neoadjuvant systemic chemotherapy; among the 19 cases in 14 patients chemoradiotherapy was also performed before or between the cycles of HIPEC. The authors reported null mortality after HIPEC while the morbidity rate was $11 \%$. In addition, 7 out of the 19 patients presented no intraoperative signs of peritoneal carcinomatosis and no imagistic signs of parenchymatous lesions at the end of the protocol were observed; negative cytology results. Therefore, they were proposed for radical surgery, and 5 of them were further submitted to radical gastrectomy; the number of HIPEC procedures performed in these cases ranging from 1 to 10 procedures (while the remaining two patients refused radical surgery). With regard to the long-term outcomes, the median overall survival after the first cycle of HIPEC was 20,3 months while the median overall survival after resection was 29 months. After a median follow up period of 18,9 months, 3 cases developed recurrent disease while the other 2 cases reported no signs of recurrence at 29 and 32 months, respectively (25).

Another interesting study from Yonemura et al was published in 2017; the study included 105 patients diagnosed with peritoneal carcinomatosis from gastric cancer. There were 53 cases who were submitted to 2 cycles of neoadjuvant HIPEC followed by interval debulking surgery and 52 cases submitted to 3 cycles of NIPS followed by debulking surgery. The authors demonstrated that in both groups the value of PCI significantly decreased after performing intraperitoneal chemotherapy while the overall rate of complete cytoreduction was $57,6 \%$ among the entire study group. In addition, the median survival rate was 19,2 months while the two-year survival rate was $41 \%$ (13).

This observation is particularly important due to the fact that, independent of the completeness of cytoreduction, the value of PCI appears to significantly influence the long-term outcomes (26-35). Therefore, in a study conducted by Glehen et al the authors demonstrated that none of the patients presenting a PCI $>12$ had survived at the three-year follow up after radical surgery and HIPEC even if complete cytoreduction had been achieved (8). In this respect, in the last decade it has been considered that debulking surgery and HIPEC should be considered as contraindications in patients presenting extended peritoneal lesions (21,30-35).

\section{Conclusions}

Neoadjuvant intraperitoneal chemotherapy delivered as HIPEC or in association with NIPS appears to provide promising results in the setting of advanced stage gastric cancer with positive cytology as well as in highly selected cases presenting peritoneal metastases. These methods have been demonstrated to be efficient in decreasing the peritoneal contamination and increasing the chances of achieving radical resections at the time of curative surgery. However, more prospective studies are still required in order to determine the best candidates for such procedures.

\section{Acknowledgements}

Not applicable. 


\section{Funding}

No funding was received.

\section{Availability of data and materials}

Not applicable.

\section{Authors' contributions}

NB, IB and MD contributed to the conception and design of the present study. CD, BS, FG, IB consulted relevant references and performed the literature data collection. IB and CS wrote the first draft of the manuscript. NB and IC revised the manuscript. All authors read and approved the final manuscript.

\section{Ethics approval and consent to participate}

Not applicable.

\section{Patient consent for publication}

Not applicable.

\section{Competing interests}

The authors declare that they have no competing interests.

\section{References}

1. Parray A, Gupta V, Chaudhari VA, Shrikhande SV and Bhandare MS: Role of intraperitoneal chemotherapy in gastric cancer. Surg Prac Sci 4: 100025, 2021.

2. Thomassen I, van Gestel YR, van Ramshorst B, Luyer MD, Bosscha K, Nienhuijs SW, Lemmens VE and de Hingh IH: Peritoneal carcinomatosis of gastric origin: A population-based study on incidence, survival and risk factors. Int J Cancer 134: 622-628, 2014.

3. Yoo CH, Noh SH, Shin DW, Choi SH and Min JS: Recurrence following curative resection for gastric carcinoma. Br J Surg 87: 236-242, 2000.

4. Sadeghi B, Arvieux C, Glehen O, Beaujard AC, Rivoire M, Baulieux J, Fontaumard E, Brachet A, Caillot JL, Faure JL, et al: Peritoneal carcinomatosis from non-gynecologic malignancies: Results of the EVOCAPE 1 multicentric prospective study. Cancer 88: 358-363, 2000.

5. Boku N; Gastrointestinal Oncology Study Group of Japan Clinical Oncology Group. Chemotherapy for metastatic disease: Review from JCOG trials. Int J Clin Oncol 13: 196-200, 2008.

6. Mezhir JJ, Shah MA, Jacks LM, Brennan MF, Coit DG and Strong VE: Positive peritoneal cytology in patients with gastric cancer: Natural history and outcome of 291 patients. Indian J Surg Oncol 2: 16-23, 2011.

7. Maeda H, Kobayashi M and Sakamoto J: Evaluation and treatment of malignant ascites secondary to gastric cancer. World $\mathrm{J}$ Gastroenterol 21: 10936-10947, 2015.

8. Glehen O, Mohamed F and Gilly FN: Peritoneal carcinomatosis from digestive tract cancer: New management by cytoreductive surgery and intraperitoneal chemohyperthermia. Lancet Oncol 5: 219-228, 2004.

9. Brenkman HJ, Päeva M, van Hillegersberg R, Ruurda JP and Haj Mohammad N: Pro-phylactic hyperthermic intraperitoneal chemotherapy (HIPEC) for gastric cancer-a systematic review. J Clin Med 8: 1685, 2019.

10. Desiderio J, Chao J, Melstrom L, Warner S, Tozzi F, Fong Y, Parisi A and Woo Y: The 30-year experience-a meta-analysis of randomised and high-quality non-randomised studies of hyperthermic intraperitoneal chemotherapy in the treatment of gastric cancer. Eur J Cancer 79: 1-14, 2017.
11. Blum Murphy M, Ikoma N, Wang X, Estrella J, Roy-Chowdhuri S, Das P, Minsky BD, Song S, Mansfield P, Ajani $\mathrm{J}$ and Badgwell B: Phase I trial of hyperthermic intraperitoneal chemoperfusion (HIPEC) with cisplatin, mitomycin, and paclitaxel in patients with gastric adenocarcinoma and associated carcinomatosis or positive cytology. Ann Surg Oncol 27: 2806-2811, 2020.

12. Chia CS, You B, Decullier E, Vaudoyer D, Lorimier G, Abboud K, Bereder JM, Arvieux C, Boschetti G and Glehen O; BIG RENAPE Group: Patients with peritoneal carcinomatosis from gastric cancer treated with cytoreductive surgery and hyperthermic intraperitoneal chemotherapy: Is cure a possibility? Ann Surg Oncol 23: 1971-1979, 2016.

13. Yonemura Y, Ishibashi H, Hirano M, Mizumoto A, Takeshita K, Noguchi K, Takao N, Ichinose M, Liu Y and Li Y: Effects of neoadjuvant laparoscopic hyperthermic intraperitoneal chemotherapy and neoadjuvant intraperitoneal/systemic chemotherapy on peritoneal metastases from gastric cancer. Ann Surg Oncol 24: 478-485, 2017.

14. Ikoma N, Chen HC, Wang X, Blum M, Estrella JS, Fournier K, Mansfield P, Ajani J and Badgwell BD: Patterns of initial recurrence in gastric adenocarcinoma in the era of preoperative therapy. Ann Surg Oncol 24: 2679-2687, 2017.

15. Ikoma N, Blum M, Chiang YJ, Estrella JS, Roy-Chowdhuri S, Fournier K, Mansfield P, Ajani J and Badgwell BD: Yield of staging laparoscopy and lavage cytology for radiologically occult peritoneal carcinomatosis of gastric cancer. Ann Surg Oncol 23: 4332-4337, 2016.

16. Newhook TE, Agnes A, Blum M, Estrella JS, Das P, Ho L, Ajani JA, Minsky BD, Mansfield P and Badgwell BD: Laparoscopic hyperthermic intraperitoneal chemotherapy is safe for patients with peritoneal metastases from gastric cancer and may lead to gastrectomy. Ann Surg Oncol 26: 1394-1400, 2019.

17. Coccolini F, Cotte E, Glehen O, Lotti M, Poiasina E, Catena F, Yonemura Y and Ansaloni L: Intraperitoneal chemotherapy in advanced gastric cancer. Meta-analysis of randomized trials. Eur J Surg Oncol 40: 12-26, 2014.

18. Yan TD, Black D, Sugarbaker PH, Zhu J, Yonemura Y, Petrou G and Morris DL: A systematic review and meta-analysis of the randomized controlled trials on adjuvant intraperitoneal chemotherapy for resectable gastric cancer. Ann Surg Oncol 14: 2702-2713, 2007.

19. Yang XJ, Huang CQ, Suo T, Mei LJ, Yang GL, Chen FL, Zhou YF, Xiong B, Yonemura Y and Li Y: Cytoreductive surgery and hyperthermic intraperitoneal chemotherapy improves survival of patients with peritoneal carcinomatosis from gastric cancer: Final results of a phase III randomized clinical trial. Ann Surg Oncol 18: 1575-1581, 2011.

20. Badgwell B: Multimodality therapy of localized gastric adenocarcinoma. J Natl Compr Canc Netw 14: 1321-1327, 2016.

21. Sugarbaker PH: Gastric cancer: Prevention and treatment of peritoneal metastases. J Cancer Metastasis Treat 4: 7, 2018.

22. Yu P, Ye Z, Dai G, Zhang Y, Huang L, Du Y and Cheng X: Neoadjuvant systemic and hyperthermic intraperitoneal chemotherapy combined with cytoreductive surgery for gastric cancer patients with limited peritoneal metastasis: A prospective cohort study. BMC Cancer 20: 1108, 2020.

23. Beeharry MK, Zhang TQ, Liu WT and Gang ZZ: Optimization of perioperative approaches for advanced and late stages of gastric cancer: Clinical proposal based on literature evidence, personal experience, and ongoing trials and research. World $\mathrm{J}$ Surg Oncol 18: 51, 2020.

24. Beeharry MK, Ni ZT, Yang ZY, Zheng YN, Feng RH, Liu WT, Yan C, Yao XX, Li C, Yan M and Zhu ZG: Study protocol of a multicenter phase III randomized controlled trial investigating the efficiency of the combination of neoadjuvant chemotherapy (NAC) and neoadjuvant laparoscopic intraperitoneal hyperthermic chemotherapy (NLHIPEC) followed by R0 gastrectomy with intraoperative HIPEC for advanced gastric cancer (AGC): Dragon II trial. BMC Cancer 20: 224, 2020.

25. Badgwell B, Blum M, Das P, Estrella J, Wang X, Ho L, Fournier K, Royal R, Mansfield P and Ajani J: Phase II trial of laparoscopic hyperthermic intraperitoneal chemoperfusion for peritoneal carcinomatosis or positive peritoneal cytology in patients with gastric adenocarcinoma. Ann Surg Oncol 24: 3338-3344, 2017.

26. Sarela AI, Miner TJ, Karpeh MS, Coit DG, Jaques DP and Brennan MF: Clinical outcomes with laparoscopic stage M1, unresected gastric adenocarcinoma. Ann Surg 243: 189-195, 2006.

27. Brenner H, Rothenbacher D and Arndt V: Epidemiology of stomach cancer. Methods Mol Biol 472: 467-477, 2009. 
28. Cappellani A, Zanghi A, Di Vita M, Zanet E, Veroux P, Cacopardo B, Cavallaro A, Piccolo G, Lo Menzo E, Murabito P and Berretta $\mathrm{M}$ : Clinical and biological markers in gastric cancer: Update and perspectives. Front Biosci (Schol Ed) 2: 403-412, 2010

29. Feingold PL, Kwong ML, Sabesan A, Sorber R and Rudloff U: Cytoreductive surgery and hyperthermic intraperitoneal chemotherapy for gastric cancer and other less common disease histologies: Is it time? J Gastrointest Oncol 7: 87-98, 2016.

30. Wu XJ, Yuan P, Li ZY, Bu ZD, Zhang LH, Wu AW, Zong XL, Li SX, Shan F, Ji X, et al: Cytoreductive surgery and hyperthermic intraperitoneal chemotherapy improves the survival of gastric cancer patients with ovarian metastasis and peritoneal dissemination. Tumor Biol 34: 463-469, 2013.

31. Yonemura Y, Kawamura T, Bandou E, Takahashi S, Sawai T and Matsuki N: Treatment of peritoneal dissemination from gastric cancer by peritonectomy and chemohyperthermic peritoneal perfusion. Br J Surg 92: 370-375, 2005

32. Takahashi T, Hagiwara A, Shimotsuma M, Sawai T and Yamaguchi T: Prophylaxis and treatment of peritoneal carcinomatosis: Intraperitoneal chemotherapy with mitomycin C bound to activated carbon particles. World J Surg 19: 565-569, 1995.
33. Bryan ML, Fitzgerald NC, Levine EA, Shen P, Stewart JH and Votanopoulos KI: Cytoreductive surgery with hyperthermic intraperitoneal chemotherapy in sarcomatosis from gastrointestinal stromal tumor. Am Surg 80: 890-895, 2014.

34. Braeuer F, Fischer I, Brammen L, Pressl G, Fuegger R, Rohregger $\mathrm{K}$ and Wundsam $\mathrm{H}$ : Outcome in patients treated with cytoreductive surgery and HIPEC for gastric cancer with peritoneal carcinomatosis. Anticancer Res 40: 2151-2156, 2020.

35. Bonnot PE, Piessen G, Kepenekian V, Decullier E, Pocard M, Menuier B, Bereder JM, Abboud K, Marchal F, Quenet F, et al: Cytoreductive surgery with or without hyperthermic intraperitoneal chemotherapy for gastric cancer with peritoneal metastases (CYTO-CHIP study): A propensity score analysis. J Clin Oncol 37: 2028-2040, 2019. 